\title{
PARTIAL ORDERS ON SEMIGROUPS OF PARTIAL TRANSFORMATIONS WITH RESTRICTED RANGE
}

\author{
KRITSADA SANGKHANAN and JINTANA SANWONG ${ }^{凶}$
}

(Received 23 August 2011)

\begin{abstract}
Let $X$ be any set and $P(X)$ the set of all partial transformations defined on $X$, that is, all functions $\alpha: A \rightarrow B$ where $A, B$ are subsets of $X$. Then $P(X)$ is a semigroup under composition. Let $Y$ be a subset of $X$. Recently, Fernandes and Sanwong defined $P T(X, Y)=\{\alpha \in P(X): X \alpha \subseteq Y\}$ and defined $I(X, Y)$ to be the set of all injective transformations in $P T(X, Y)$. Hence $P T(X, Y)$ and $I(X, Y)$ are subsemigroups of $P(X)$. In this paper, we study properties of the so-called natural partial order $\leq$ on $P T(X, Y)$ and $I(X, Y)$ in terms of domains, images and kernels, compare $\leq$ with the subset order, characterise the meet and join of these two orders, then find elements of $P T(X, Y)$ and $I(X, Y)$ which are compatible. Also, the minimal and maximal elements are described.
\end{abstract}

2010 Mathematics subject classification: primary 20M20.

Keywords and phrases: partial transformation semigroups, natural order, compatibility, maximal and minimal elements.

\section{Introduction}

The natural partial order on a semigroup has been developed in a number of steps. In the terminology of Clifford and Preston [1], a band $B$ is a semigroup in which every element is an idempotent. On such a semigroup there is a natural (partial) order defined by the rule

$$
e \leq f \quad \text { if and only if } \quad e=e f=f e .
$$

If the partial order $\leq$ is compatible with the multiplication in $B$, in the sense that $e \leq f$ implies that $e g \leq f g$ and $g e \leq g f$ for all $g \in B$, we shall say that $B$ is a naturally ordered band. In 1966, Howie [4] described the structure of naturally ordered bands.

The first author thanks the Development and Promotion of Science and Technology talents project, Thailand, for its financial support. He also thanks the Graduate School, Chiang Mai University, Chiangmai, Thailand, for its financial support that he received during the preparation of this paper. The second author thanks the National Research University Project under the Office of the Higher Education Commission, Thailand, for its financial support.

(C) 2012 Australian Mathematical Publishing Association Inc. 0004-9727/2012 \$16.00 
In the year 1952, Vagner [13] defined the natural order on an inverse semigroup $S$ by

$$
a \leq b \quad \text { if and only if } \quad a=e b \quad \text { for some } e \in E(S) .
$$

About thirty years later, Hartwig [3] and Nambooripad [9] independently discovered the generalisation of the above order. They defined it on a regular semigroup $S$ by

$$
a \leq b \quad \text { if and only if } \quad a=e b=b f \quad \text { for some } e, f \in E(S) .
$$

In general $\leq$ is not compatible with multiplication on $S$. In 1986, the natural order on a regular semigroup was further extended to any semigroup $S$ by Mitsch [8]. He defined

$$
a \leq b \quad \text { if and only if } \quad a=x b=b y, x a=a \quad \text { for some } x, y \in S^{1} .
$$

The partial transformation semigroup on the set $X$, denoted $P(X)$, is the set of all functions from a subset of $X$ into $X$, with the operation of composition. In addition, the semigroups $T(X)$ and $I(X)$ are defined by

$$
\begin{aligned}
T(X) & =\{\alpha \in P(X): \text { dom } \alpha=X\}, \\
I(X) & =\{\alpha \in P(X): \alpha \text { is injective }\} .
\end{aligned}
$$

These semigroups $T(X)$ and $I(X)$ are called the full transformation semigroup and the symmetric inverse semigroup, respectively. It is well known that $P(X)$ and $T(X)$ are regular and that $I(X)$ is an inverse semigroup.

In 1986, Kowol and Mitsch [6] studied the full transformation semigroup $T(X)$ on the set $X$ with respect to the natural partial order which is defined by (1.1). They characterised this order in terms of images and kernels. They also described the maximal, minimal and covering elements. Additionally, they studied lower and upper bounds for two transformations.

In 2003, Marques-Smith and Sullivan [7] studied the natural partial order on $P(X)$ and $T(X)$. They also investigated the orders $\subseteq, \Omega^{\prime}$ and $\Omega$ on $P(X)$ which are defined as follows.

(i) $\alpha \subseteq \beta$ if and only if $\operatorname{dom} \alpha \subseteq \operatorname{dom} \beta$ and $x \alpha=x \beta$ for all $x \in \operatorname{dom} \alpha$.

(ii) $(\alpha, \beta) \in \Omega^{\prime}$ if and only if $X \alpha \subseteq X \beta$, $\operatorname{dom} \alpha \subseteq \operatorname{dom} \beta$ and

$$
\alpha \beta^{-1} \cap(\operatorname{dom} \alpha \times \operatorname{dom} \alpha) \subseteq \alpha \alpha^{-1} .
$$

(iii) $(\alpha, \beta) \in \Omega$ if and only if $(\alpha, \beta) \in \Omega^{\prime}$ and

$$
\beta \beta^{-1} \cap(\operatorname{dom} \alpha \times \operatorname{dom} \alpha) \subseteq \alpha \alpha^{-1} .
$$

They proved that $\Omega=\subseteq \circ \leq$ is a join of $\leq$ and $\subseteq$. Moreover, they found elements of $P(X)$ and $T(X)$ which are compatible under $\leq$ and $\subseteq$. Also, the maximal and minimal elements were described. Recently in [11], the authors considered these orders on $I(X)$ and found that $\Omega=\Omega^{\prime}$ and $\subseteq$ is always properly contained in $\Omega$ for $|X|>1$. 
Let $Y$ be a nonempty subset of $X$. We consider a subsemigroup of $T(X)$ defined by

$$
T(X, Y)=\{\alpha \in T(X): X \alpha \subseteq Y\}
$$

where $X \alpha$ denotes the range of $\alpha$. In 1975, Symons [12] introduced and studied the semigroup $T(X, Y)$. He described all the automorphisms of $T(X, Y)$ and found that the most difficult case occurs when $|Y|=2$. He also determined when $T\left(X_{1}, Y_{1}\right)$ is isomorphic to $T\left(X_{2}, Y_{2}\right)$. In 2008, Sanwong and Sommanee [10] obtained the largest regular subsemigroup of $T(X, Y)$ and a class of its maximal inverse subsemigroups. Further, they characterised the Green's relations on $T(X, Y)$.

In [2], Fernandes and Sanwong introduced the partial transformation semigroup with restricted range as follows.

Let $Y$ be a subset of $X$. They considered the semigroup $P T(X, Y)$ and $I(X, Y)$ defined by

$$
P T(X, Y)=\{\alpha \in P(X): X \alpha \subseteq Y\} \quad \text { and } \quad I(X, Y)=I(X) \cap P T(X, Y) .
$$

Clearly, $P T(X, X)=P(X), T(X, X)=T(X), I(X, X)=I(X)$ and $P T(X, \emptyset)=I(X, \emptyset)=$ $\{\emptyset\}$. Moreover, they proved that $P F=\{\alpha \in P T(X, Y): X \alpha=Y \alpha\}$ is the largest regular subsemigroup of $P T(X, Y)$ and that $I(Y)$ is the largest regular subsemigroup of $I(X, Y)$.

In this paper, we characterise $\leq$ and $\subseteq$ on $P T(X, Y)$ and $I(X, Y)$, and describe the meet and join of $\leq$ and $\subseteq$. Then we compare $\leq, \subseteq$ with other partial orders and find elements of $P T(X, Y), I(X, Y)$ which are compatible with $\leq$. Also, the minimal and maximal elements of $P T(X, Y)$ with respect to $\leq$ are obtained.

\section{Preliminary notations and results}

In this section, we give some notations and results which are used in this paper.

Recall that the natural partial order on any semigroup $S$ is defined by $a \leq b \quad$ if and only if $a=x b=b y, \quad x a=a \quad$ for some $x, y \in S^{1}$, or equivalently

$$
a \leq b \quad \text { if and only if } \quad a=w b=b z, \quad a z=a \quad \text { for some } w, z \in S^{1} .
$$

In this paper, we use (2.1) to define the partial order on the semigroup $S=P T(X, Y)$ or $I(X, Y)$; that is, for each $\alpha, \beta \in S$

$$
\alpha \leq \beta \quad \text { if and only if } \quad \alpha=\gamma \beta=\beta \mu, \quad \alpha=\alpha \mu \quad \text { for some } \gamma, \mu \in S^{1} .
$$

We note that if $Y \subsetneq X$, then $P T(X, Y)$ and $I(X, Y)$ have no identity elements. Thus, in this case $P T(X, Y)^{1} \neq P T(X, Y)$ and $I(X, Y)^{1} \neq I(X, Y)$.

In this paper, the kernel equivalence of $\alpha \in P T(X, Y)$, ker $\alpha$, is defined by

$$
(x, y) \in \operatorname{ker} \alpha \quad \text { if and only if } \quad x \alpha=y \alpha .
$$

To describe the natural partial order on $P T(X, Y)$ and $I(X, Y)$, we need the following two lemmas which first appeared in [2]. For the sake of completeness, we present the proofs here. 
Lemma 2.1. Let $\alpha, \beta \in P T(X, Y)$. Then

$$
\operatorname{dom} \alpha \subseteq \operatorname{dom} \beta \quad \text { and } \quad \operatorname{ker} \beta \cap(\operatorname{dom} \beta \times \operatorname{dom} \alpha) \subseteq \operatorname{ker} \alpha
$$

if and only if $\alpha=\beta \gamma$ for some $\gamma \in P T(X, Y)$.

Proof. If $\alpha=\beta \gamma$, for some $\gamma \in P T(X, Y)$, then it is clear that $\operatorname{dom} \alpha \subseteq \operatorname{dom} \beta$. On the other hand, consider $(a, b) \in \operatorname{ker} \beta \cap(\operatorname{dom} \beta \times \operatorname{dom} \alpha)$. Then we have $a \beta=b \beta$. Since $b \in \operatorname{dom} \alpha=\operatorname{dom} \beta \gamma$, it follows that $b \beta \gamma$ exists, whence $b \beta \gamma=(b \beta) \gamma=(a \beta) \gamma=a \beta \gamma$, and so we also obtain $a \in \operatorname{dom} \beta \gamma=\operatorname{dom} \alpha$. Moreover, $a \alpha=(a \beta) \gamma=(b \beta) \gamma=b \alpha$, whence $(a, b) \in \operatorname{ker} \alpha$. Thus $\operatorname{ker} \beta \cap(\operatorname{dom} \beta \times \operatorname{dom} \alpha) \subseteq \operatorname{ker} \alpha$.

Conversely, assume that the conditions hold. Let $x \in(\operatorname{dom} \alpha) \beta$. Then $a \beta=$ $x$, for some $a \in \operatorname{dom} \alpha$. Notice that if $b \in \operatorname{dom} \beta$ is also such that $b \beta=x$ then $(b, a) \in \operatorname{ker} \beta \cap(\operatorname{dom} \beta \times \operatorname{dom} \alpha)$. Hence, by the hypothesis, we have $b \alpha=a \alpha$ (and, in particular, $b \in \operatorname{dom} \alpha$ ). Thus, we consider the transformation $\gamma \in P T(X, Y)$ with $\operatorname{dom} \gamma=(\operatorname{dom} \alpha) \beta$ defined by, for each $x \in(\operatorname{dom} \alpha) \beta, x \gamma=a \alpha$, for some $a \in \operatorname{dom} \alpha$ such that $a \beta=x$. Hence, $\alpha=\beta \gamma$, as required.

Lemma 2.2. Let $\alpha, \beta \in I(X, Y)$. Then $\operatorname{dom} \alpha \subseteq \operatorname{dom} \beta$ if and only if $\alpha=\beta \gamma$ for some $\gamma \in I(X, Y)$.

Proof. If $\alpha=\beta \gamma$, for some $\gamma \in I(X, Y)$, then clearly, $\operatorname{dom} \alpha \subseteq \operatorname{dom} \beta$. Conversely, suppose that $\operatorname{dom} \alpha \subseteq \operatorname{dom} \beta$. Then we can write $\alpha=\left(\begin{array}{l}x_{i} \\ a_{i}\end{array}\right)$ and $\beta=\left(\begin{array}{l}x_{i} x_{j} \\ b_{i} b_{j}\end{array}\right)$, where $\left\{a_{i}, b_{i}, b_{j}\right\} \subseteq Y$. Now define $\gamma=\left(\begin{array}{c}b_{i} \\ a_{i}\end{array}\right) \in I(X, Y)$; we have $\alpha=\beta \gamma$, as required.

We have the following simple result on $P T(X, Y)$ which will be used throughout the paper.

Lemma 2.3. If $A \subseteq B$, then $A \alpha \subseteq B \alpha$ for all $\alpha \in P T(X, Y)$.

The following convenient notation will be used: given $\alpha \in P T(X, Y)$, we write

$$
\alpha=\left(\begin{array}{l}
X_{i} \\
a_{i}
\end{array}\right)
$$

and take as understood that the subscript $i$ belongs to some (unmentioned) index set $I$, that the abbreviation $\left\{a_{i}\right\}$ denotes $\left\{a_{i}: i \in I\right\}$, and that $X \alpha=\left\{a_{i}\right\}$ and $a_{i} \alpha^{-1}=X_{i}$.

\section{Partial orders}

If we regard $\alpha, \beta \in P T(X, Y)$ as subsets of $X \times Y$, it is easy to see that

$\alpha \subseteq \beta \quad$ if and only if $\quad \operatorname{dom} \alpha \subseteq \operatorname{dom} \beta \quad$ and $\quad x \alpha=x \beta \quad$ for all $x \in \operatorname{dom} \alpha$.

We also have $\subseteq$ is a partial order on $P T(X, Y)$ and $\emptyset \subseteq \alpha$ for all $\alpha \in P T(X, Y)$. Similarly, for $\alpha, \beta \in I(X, Y)$ we have $\alpha \subseteq \beta$ if and only if $\operatorname{dom} \alpha \subseteq \operatorname{dom} \beta$ and $x \alpha=x \beta$ for all $x \in \operatorname{dom} \alpha$; and $\subseteq$ is a partial order on $I(X, Y)$ with $\emptyset \subseteq \alpha$ for all $\alpha \in I(X, Y)$.

To characterise the natural partial order $\leq$ and the subset order $\subseteq$ on $P T(X, Y)$ and $I(X, Y)$, we begin with the following lemma. 
Lemma 3.1. Let $\alpha, \beta \in P T(X, Y)$. Then $X \alpha \subseteq Y \beta$ if and only if $\alpha=\gamma \beta$ for some $\gamma \in$ $P T(X, Y)$.

Proof. Assume that $X \alpha \subseteq Y \beta$. Then for each $x \in \operatorname{dom} \alpha$, we have $x \alpha=y \beta$ for some $y \in Y$, which implies that $y \in Y \cap(x \alpha) \beta^{-1} \neq \emptyset$. Choose $d_{x} \in Y \cap(x \alpha) \beta^{-1}$, so $d_{x} \in Y$ and $d_{x} \beta=x \alpha$. Now, define $\gamma: \operatorname{dom} \alpha \rightarrow Y$ by $x \gamma=d_{x}$ for all $x \in \operatorname{dom} \alpha$. Thus $\gamma \in P T(X, Y)$ and $x \gamma \beta=(x \gamma) \beta=d_{x} \beta=x \alpha$ for all $x \in \operatorname{dom} \alpha$. In addition, we obtain $\operatorname{dom} \gamma \beta=(\operatorname{im} \gamma \cap \operatorname{dom} \beta) \gamma^{-1}=(\operatorname{im} \gamma) \gamma^{-1}=\operatorname{dom} \gamma=\operatorname{dom} \alpha$. The converse is clearly true since $X \alpha=X \gamma \beta=(X \gamma) \beta \subseteq Y \beta$.

In the proof of Lemma 3.1, if $\alpha, \beta \in I(X, Y)$, then $\gamma$ is also in $I(X, Y)$. Hence, we obtain the following lemma immediately.

Lemma 3.2. Suppose that $\alpha, \beta \in I(X, Y)$. Then $X \alpha \subseteq Y \beta$ if and only if $\alpha=\gamma \beta$ for some $\gamma \in I(X, Y)$.

By Lemmas 3.1 and 2.1, we obtain the characterisation of $\leq$ on $P T(X, Y)$ as follows.

Theorem 3.3. Let $\alpha, \beta \in P T(X, Y)$. Then $\alpha \leq \beta$ if and only if $\alpha=\beta$ or the following statements hold.

(1) $X \alpha \subseteq Y \beta$.

(2) $\operatorname{dom} \alpha \subseteq \operatorname{dom} \beta$ and $\operatorname{ker} \beta \cap(\operatorname{dom} \beta \times \operatorname{dom} \alpha) \subseteq \operatorname{ker} \alpha$.

(3) For each $x \in \operatorname{dom} \beta$, if $x \beta \in X \alpha$, then $x \in \operatorname{dom} \alpha$ and $x \alpha=x \beta$.

Proof. Suppose that $\alpha \leq \beta$. Then there exist $\gamma, \mu \in P T(X, Y)^{1}$ such that $\alpha=\gamma \beta=\beta \mu$ and $\alpha=\alpha \mu$. If $\gamma=1$ or $\mu=1$, then $\alpha=\beta$. If $\gamma, \mu \in P T(X, Y)$, then conditions (1) and (2) hold by Lemmas 3.1 and 2.1. If $x \in \operatorname{dom} \beta$ and $x \beta \in X \alpha$, then $x \beta=y \alpha$ for some $y \in X$, and thus

$$
x \beta=y \alpha=y \alpha \mu=x \beta \mu=x \alpha .
$$

Therefore, $x \in \operatorname{dom} \alpha$ and $x \alpha=x \beta$. Conversely, assume that the conditions (1)-(3) hold. Again by Lemmas 3.1 and 2.1, there exist $\gamma, \mu \in P T(X, Y)$ such that $\alpha=\gamma \beta=\beta \mu$. Now, we prove that $\operatorname{im} \alpha \subseteq \operatorname{dom} \mu$, by letting $y \in \operatorname{im} \alpha$. Then there is $x \in \operatorname{dom} \alpha$ such that $x \alpha=y$. Since $\alpha=\gamma \beta$, we have $y=x \alpha=x \gamma \beta$. By condition (3), $x \gamma \in \operatorname{dom} \alpha$ and $x \gamma \alpha=x \gamma \beta$. Thus $x \gamma \beta=x \gamma \alpha=x \gamma \beta \mu=y \mu$, which implies that $y \in \operatorname{dom} \mu$. Therefore, $\operatorname{im} \alpha \subseteq \operatorname{dom} \mu$. Hence

$$
\operatorname{dom} \alpha \mu=(\operatorname{im} \alpha \cap \operatorname{dom} \mu) \alpha^{-1}=(\operatorname{im} \alpha) \alpha^{-1}=\operatorname{dom} \alpha .
$$

For each $x \in \operatorname{dom} \alpha, x \alpha=x \gamma \beta$. Again by condition (3), $x \gamma \in \operatorname{dom} \alpha$ and $x \gamma \alpha=x \gamma \beta$. Thus

$$
x \alpha=x \gamma \beta=x \gamma \alpha=x \gamma \beta \mu=x \alpha \mu \text {. }
$$

Therefore, $\alpha=\alpha \mu$.

Notice that $\emptyset \leq \alpha$ for all $\alpha \in P T(X, Y)$.

Now, we consider the semigroup $I(X, Y)$. 
Theorem 3.4. Let $\alpha, \beta \in I(X, Y)$. Then $\alpha \leq \beta$ if and only if $\alpha=\beta$ or the following statements hold.

(1) $X \alpha \subseteq Y \beta$.

(2) $\operatorname{dom} \alpha \subseteq \operatorname{dom} \beta$.

(3) For each $x \in \operatorname{dom} \beta$, if $x \beta \in X \alpha$, then $x \in \operatorname{dom} \alpha$ and $x \alpha=x \beta$.

Proof. Since $\alpha, \beta \in I(X, Y) \subseteq P T(X, Y)$ and $\alpha \leq \beta$, we have by Theorem 3.3 that $\alpha=\beta$ or the conditions (1)-(3) hold.

Using Lemmas 3.2 and 2.2 in the proof as given for Theorem 3.3, we obtain the converse of the theorem.

Clearly, if $\rho$ and $\sigma$ are partial orders on $X$ then the intersection of $\rho$ and $\sigma, \rho \cap \sigma$, is also a partial order on $X$. Now we have three partial orders on $P T(X, Y)$, namely, $\leq, \subseteq$ and $\leq \cap \subseteq$. The following lemma shows that if $|Y| \geq 2$, then $\subseteq \backslash \leq$ and $\leq \backslash \subseteq$ on $P T(X, Y)$ are nonempty. Consequently, the meet of $\leq$ and $\subseteq$ cannot equal $\leq$ or $\subseteq$, so these three partial orders are different.

Lemma 3.5. If $|X| \geq 2$, then the following statements hold on $P T(X, Y)$.

(1) $\subseteq \backslash \leq$ is nonempty.

(2) $\leq \backslash \subseteq$ is nonempty if and only if $|Y| \geq 2$.

Proof. (1) Let $a \in X, b \in Y$ be such that $a \neq b$ and let

$$
\alpha=\left(\begin{array}{l}
a \\
b
\end{array}\right), \quad \beta=\left(\begin{array}{c}
\{a, b\} \\
b
\end{array}\right) .
$$

Then $\alpha \subseteq \beta$, and $b \beta=b \in X \alpha$ while $b \notin \operatorname{dom} \alpha$. Hence $\alpha \not \leq \beta$; that is, $\subseteq \backslash \leq$ is nonempty.

(2) Suppose that $|Y| \geq 2$. Let $a, b \in Y \subseteq X$ be such that $a \neq b$, and define

$$
\alpha=\left(\begin{array}{c}
\{a, b\} \\
b
\end{array}\right), \quad \beta=\left(\begin{array}{ll}
a & b \\
a & b
\end{array}\right) .
$$

Then $\alpha \nsubseteq \beta$. We see that $X \alpha=\{b\} \subseteq\{a, b\}=Y \beta$ and $\operatorname{dom} \alpha \subseteq \operatorname{dom} \beta$. Also,

$$
\operatorname{ker} \beta \cap(\operatorname{dom} \beta \times \operatorname{dom} \alpha)=\{(a, a),(b, b)\} \subseteq\{(a, a),(b, b),(a, b),(b, a)\}=\operatorname{ker} \alpha,
$$

and $b \beta=b \in X \alpha$ implies $b \in \operatorname{dom} \alpha$ and $b \alpha=b \beta$. Hence $\alpha \leq \beta$; that is, $\leq \backslash \subseteq$ is nonempty.

Conversely, suppose that $|Y|=1$. Let $\alpha, \beta \in P T(X, Y)$ be such that $\alpha \leq \beta$. Then $\operatorname{dom} \alpha \subseteq \operatorname{dom} \beta$ and, if $x \in \operatorname{dom} \alpha$, we have $x \in \operatorname{dom} \beta$ and $x \alpha=x \beta$ since $|Y|=1$. Thus $\alpha \subseteq \beta$, and hence $\leq \subseteq \subseteq$, which implies that $\leq \backslash \subseteq$ is empty.

We now intend to determine their join.

Lemma 3.6. The join $\subseteq$ $\leq \leq$ is a partial order on $P T(X, Y)$. 
Proof. It is clear that $\subseteq \circ \leq$ is reflexive. Let $\alpha, \beta \in P T(X, Y)$ be such that $(\alpha, \beta),(\beta, \alpha) \in \subseteq \circ \leq$. Then there exist $\gamma, \mu \in P T(X, Y)$ such that $\alpha \subseteq \gamma \leq \beta$ and $\beta \subseteq$ $\mu \leq \alpha$. Hence

$$
\operatorname{dom} \alpha \subseteq \operatorname{dom} \gamma \subseteq \operatorname{dom} \beta \subseteq \operatorname{dom} \mu \subseteq \operatorname{dom} \alpha .
$$

Therefore, $\operatorname{dom} \alpha=\operatorname{dom} \beta$. Now, we prove that $\alpha=\beta$. For each $x \in \operatorname{dom} \alpha, x \alpha=$ $x \gamma \in X \gamma \subseteq Y \beta$ since $\alpha \subseteq \gamma \leq \beta$. Hence $x \gamma=y \beta$ for some $y \in Y$, and then $y \beta \in X \gamma$, which implies that $y \in \operatorname{dom} \gamma$ and $y \beta=y \gamma$, since $\gamma \leq \beta$. Since $\beta \subseteq \mu$, we obtain $y \beta=y \mu \in X \mu \subseteq Y \alpha$, so $y \mu=z \alpha$ for some $z \in Y$. Thus $z \alpha \in X \mu$, from which it follows that $z \in \operatorname{dom} \mu$ and $z \alpha=z \mu$ since $\mu \leq \alpha$. Hence

$$
z \alpha=y \mu=y \beta=x \gamma=x \alpha .
$$

This means that $(x, z) \in \operatorname{ker} \alpha \cap(\operatorname{dom} \alpha \times \operatorname{dom} \mu) \subseteq \operatorname{ker} \mu$, whence $x \mu=z \mu$. Since $\beta \subseteq \mu$, we obtain $x \beta=x \mu$. Hence

$$
x \beta=x \mu=z \mu=z \alpha=x \alpha .
$$

Thus $\alpha=\beta$, and therefore $\subseteq \circ \leq$ is antisymmetric.

Next, we prove the transitivity of $\subseteq 0 \leq$. Let $(\alpha, \gamma),(\gamma, \beta) \in \subseteq 0 \leq$. Then there are $\mu, \xi \in P T(X, Y)$ such that $\alpha \subseteq \mu \leq \gamma$ and $\gamma \subseteq \xi \leq \beta$. Thus

$$
\operatorname{dom} \alpha \subseteq \operatorname{dom} \mu \subseteq \operatorname{dom} \gamma \subseteq \operatorname{dom} \xi \subseteq \operatorname{dom} \beta,
$$

and

$$
X \alpha \subseteq X \mu \subseteq Y \gamma \subseteq X \gamma \subseteq X \xi \subseteq Y \beta
$$

Hence we can write

$$
\alpha=\left(\begin{array}{c}
A_{i} \\
x_{i}
\end{array}\right), \quad \beta=\left(\begin{array}{ll}
B_{i} & B_{j} \\
x_{i} & x_{j}
\end{array}\right),
$$

where $\bigcup A_{i} \subseteq\left(\bigcup B_{i}\right) \cup\left(\bigcup B_{j}\right)$ and $B_{i} \cap Y \neq \emptyset$ for all $i$. Let

$$
J_{i}=\left\{j \in J: B_{j} \cap A_{i} \neq \emptyset\right\} \quad \text { and } \quad C_{i}=\bigcup\left\{B_{j}: j \in J_{i}\right\} .
$$

Define

$$
\theta=\left(\begin{array}{c}
A_{i} \cup B_{i} \cup C_{i} \\
x_{i}
\end{array}\right) .
$$

To see that $\theta$ is well defined, let $i_{1}, i_{2} \in I$ be such that $i_{1} \neq i_{2}$ and suppose that there exists $j \in J_{i_{1}} \cap J_{i_{2}}$. Then $B_{j} \cap A_{i_{1}} \neq \emptyset \neq B_{j} \cap A_{i_{2}}$. Let $x \in B_{j} \cap A_{i_{1}}$ and $y \in$ $B_{j} \cap A_{i_{2}}$. Hence $x \beta=x_{j}=y \beta$ and $x, y \in \operatorname{dom} \alpha \subseteq \operatorname{dom} \beta$. Since $\xi \leq \beta$ and $(x, y) \in$ $\operatorname{ker} \beta \cap(\operatorname{dom} \beta \times \operatorname{dom} \xi) \subseteq \operatorname{ker} \xi$, we obtain $x \xi=y \xi$, from which it follows that $x \gamma=y \gamma$ since $\gamma \subseteq \xi$. Using the condition $\alpha \subseteq \mu \leq \gamma$, we obtain $x \mu=y \mu$ and $x \alpha=y \alpha$. Thus $x_{i_{1}}=x \alpha=y \alpha=x_{i_{2}}$, which is a contradiction. Hence $J_{i_{1}} \cap J_{i_{2}}=\emptyset$, which implies that $\theta$ is well defined. It is clear that $\alpha \subseteq \theta$. Also, by the definition of $\theta$, we see that $\theta$ and $\beta$ satisfy conditions (1)-(3) of Theorem 3.3, and thus $\theta \leq \beta$. Therefore, $(\alpha, \beta) \in \subseteq \circ \leq$. 
Since $\subseteq, \leq$ and $\subseteq \circ \leq$ are partial orders on $P T(X, Y)$, we obtain the following theorem immediately.

Theorem 3.7. The partial order $\subseteq$ o $\leq$ is the join of $\subseteq$ and $\leq$ on $P T(X, Y)$.

Since $\subseteq \backslash \leq$ and $\leq \backslash \subseteq$ on $P T(X, Y)$ are nonempty, the join of $\subseteq$ and $\leq$ is equal to neither $\subseteq$ nor $\leq$ when $|Y| \geq 2$, so we now have four different nontrivial partial orders on $P T(X, Y)$, namely $\subseteq \cap \leq, \subseteq, \leq$ and $\subseteq \circ \leq$.

\section{Comparable partial orders}

Recall that in [7] the authors defined the partial orders $\Omega^{\prime}$ and $\Omega$ on $P(X)$ as follows, where $\alpha, \beta \in P(X)$.

(i) $(\alpha, \beta) \in \Omega^{\prime}$ if and only if $X \alpha \subseteq X \beta$, dom $\alpha \subseteq \operatorname{dom} \beta$ and

$$
\alpha \beta^{-1} \cap(\operatorname{dom} \alpha \times \operatorname{dom} \alpha) \subseteq \alpha \alpha^{-1} .
$$

(ii) $(\alpha, \beta) \in \Omega$ if and only if $(\alpha, \beta) \in \Omega^{\prime}$ and

$$
\beta \beta^{-1} \cap(\operatorname{dom} \alpha \times \operatorname{dom} \alpha) \subseteq \alpha \alpha^{-1} .
$$

Since $P T(X, Y)$ is a subset of $P(X)$, we see that the relations $\Omega^{\prime}$ and $\Omega$ are also partial orders on $P T(X, Y)$ and $\Omega \subseteq \Omega^{\prime}$. In [7], the authors showed that $\Omega$ is a join and $\Omega^{\prime}$ is an upper bound of $\subseteq$ and $\leq$ on $P(X)$. By the following theorem, $\Omega$ is an upper bound of $\subseteq$ and $\leq$ on $P T(X, Y)$.

Theorem 4.1. The partial order $\Omega$ is an upper bound of $\subseteq$ and $\leq$ on $P T(X, Y)$. Consequently, $\subseteq \circ \leq$ is contained in $\Omega$.

Proof. Let $\alpha, \beta \in P T(X, Y)$. If $\alpha \subseteq \beta$, then $X \alpha \subseteq X \beta$ and $\operatorname{dom} \alpha \subseteq \operatorname{dom} \beta$. For $(x, y) \in$ $\alpha \beta^{-1} \cap(\operatorname{dom} \alpha \times \operatorname{dom} \alpha)$, we have $x \alpha=y \beta$. Thus $x \alpha=y \beta=y \alpha$ since $\alpha \subseteq \beta$, which implies that $(x, y) \in \alpha \alpha^{-1}$. Similarly, we can show that

$$
\beta \beta^{-1} \cap(\operatorname{dom} \alpha \times \operatorname{dom} \alpha) \subseteq \alpha \alpha^{-1} .
$$

Hence $(\alpha, \beta) \in \Omega$.

Now, if $\alpha \leq \beta$, then $X \alpha \subseteq Y \beta \subseteq X \beta$, dom $\alpha \subseteq \operatorname{dom} \beta$ and $\operatorname{ker} \beta \cap(\operatorname{dom} \beta \times \operatorname{dom} \alpha) \subseteq$ ker $\alpha$. Let

$$
(x, y) \in \alpha \beta^{-1} \cap(\operatorname{dom} \alpha \times \operatorname{dom} \alpha) .
$$

Then $x, y \in \operatorname{dom} \alpha$ and $x \alpha=y \beta$, which implies that $y \beta \in X \alpha$. Since $\alpha \leq \beta$, we have $y \alpha=y \beta$. Hence $x \alpha=y \alpha$, from which it follows that $(x, y) \in \alpha \alpha^{-1}$. Therefore, $(\alpha, \beta) \in \Omega^{\prime}$. Let

$$
(p, q) \in \beta \beta^{-1} \cap(\operatorname{dom} \alpha \times \operatorname{dom} \alpha) .
$$

Then $p, q \in \operatorname{dom} \alpha \subseteq \operatorname{dom} \beta$ and $p \beta=q \beta$, which implies that

$$
(p, q) \in \operatorname{ker} \beta \cap(\operatorname{dom} \beta \times \operatorname{dom} \alpha) \subseteq \operatorname{ker} \alpha .
$$

Thus $p \alpha=q \alpha$ and $(p, q) \in \alpha \alpha^{-1}$. Therefore, $(\alpha, \beta) \in \Omega$. 
The following two lemmas characterise when $\Omega=\subseteq \circ \leq$ and $\Omega^{\prime}=\Omega$ on $P T(X, Y)$.

Lemma 4.2. The partial order $\Omega=\subseteq \circ \leq$ on $P T(X, Y)$ if and only if $Y=X$ or $|Y|=1$.

Proof. If $Y=X$, then $\Omega=\subseteq \circ \leq$ on $P T(X, X)=P(X)$ by [7, Theorem 7]. Assume that $|Y|=1$ and let $(\alpha, \beta) \in \Omega$. Then $\operatorname{dom} \alpha \subseteq \operatorname{dom} \beta$. If $\alpha=\emptyset$, then $\alpha \subseteq \beta$, but if $\alpha \neq \emptyset$, then for each $x \in \operatorname{dom} \alpha$ it is true that $x \alpha=x \beta$, since $|Y|=1$. Hence $\alpha \subseteq \beta$, and thus $\alpha \subseteq \beta \leq \beta$, which implies that $(\alpha, \beta) \in \subseteq \circ \leq$.

Conversely, suppose that $Y \subsetneq X$ and $|Y| \geq 2$. Then there are three distinct elements $x, y, z$ such that $x \in X \backslash Y$ and $y, z \in Y$. Define $\alpha, \beta$ by

$$
\alpha=\left(\begin{array}{l}
y \\
y
\end{array}\right), \quad \beta=\left(\begin{array}{ll}
x & y \\
y & z
\end{array}\right) .
$$

Then $\alpha, \beta \in P T(X, Y), X \alpha \subseteq X \beta$, $\operatorname{dom} \alpha \subseteq \operatorname{dom} \beta$,

$$
\alpha \beta^{-1} \cap(\operatorname{dom} \alpha \times \operatorname{dom} \alpha)=\emptyset \subseteq \alpha \alpha^{-1}
$$

and

$$
\beta \beta^{-1} \cap(\operatorname{dom} \alpha \times \operatorname{dom} \alpha)=\{(y, y)\}=\alpha \alpha^{-1} .
$$

Hence $(\alpha, \beta) \in \Omega$. Assume that $(\alpha, \beta) \in \subseteq \circ \leq$. Then there is $\gamma \in P T(X, Y)$ such that $\alpha \subseteq \gamma \leq \beta$. If $\gamma=\beta$, then $y=y \alpha=y \gamma=y \beta=z$, which is a contradiction. Hence $\gamma \neq \beta$ and $X \gamma \subseteq Y \beta$. Thus $\{y\}=X \alpha \subseteq X \gamma \subseteq Y \beta=\{z\}$, which is also a contradiction. Therefore, $(\alpha, \beta) \notin \subseteq \circ \leq$, which implies that $\Omega \backslash \subseteq \circ \leq$ is nonempty.

Lemma 4.3. The partial order $\Omega^{\prime}=\Omega$ on $P T(X, Y)$ if and only if $|Y| \leq 2$.

Proof. Suppose that $|Y| \leq 2$ and let $(\alpha, \beta) \in \Omega^{\prime}$. Then $X \alpha \subseteq X \beta$, $\operatorname{dom} \alpha \subseteq \operatorname{dom} \beta$ and

$$
\alpha \beta^{-1} \cap(\operatorname{dom} \alpha \times \operatorname{dom} \alpha) \subseteq \alpha \alpha^{-1} \text {. }
$$

Let

$$
(x, y) \in \beta \beta^{-1} \cap(\operatorname{dom} \alpha \times \operatorname{dom} \alpha) .
$$

Then $x \beta=y \beta$ and $x, y \in \operatorname{dom} \alpha$. If $x \alpha=y \alpha$, then $(x, y) \in \alpha \alpha^{-1}$. However, if $x \alpha \neq$ $y \alpha$, then $x \alpha=y \beta$ or $y \alpha=x \beta$ since $|Y| \leq 2$. Hence $(x, y)$ or $(y, x) \in \alpha \beta^{-1} \cap(\operatorname{dom} \alpha \times$ $\operatorname{dom} \alpha) \subseteq \alpha \alpha^{-1}$, and so $(x, y) \in \alpha \alpha^{-1}$. Therefore, $\beta \beta^{-1} \cap(\operatorname{dom} \alpha \times \operatorname{dom} \alpha) \subseteq \alpha \alpha^{-1}$.

Conversely, assume that $|Y| \geq 3$. If $Y=X$, then $\Omega^{\prime} \backslash \Omega$ is nonempty by [7, p. 110]. If $Y \subsetneq X$, then we suppose $\{s, t, u, v\} \subseteq X$ such that $s, t, u \in Y$, and define

$$
\alpha=\left(\begin{array}{ll}
s & t \\
s & t
\end{array}\right), \quad \beta=\left(\begin{array}{ccc}
\{s, t\} & u & v \\
u & s & t
\end{array}\right) .
$$

Therefore, $\alpha, \beta \in P T(X, Y), X \alpha \subseteq X \beta$, dom $\alpha \subseteq \operatorname{dom} \beta$ and

$$
\alpha \beta^{-1} \cap(\operatorname{dom} \alpha \times \operatorname{dom} \alpha)=\emptyset \subseteq \alpha \alpha^{-1} .
$$

Hence $(\alpha, \beta) \in \Omega^{\prime}$. Since

$$
\beta \beta^{-1} \cap(\operatorname{dom} \alpha \times \operatorname{dom} \alpha)=\{(s, s),(t, t),(s, t),(t, s)\} \nsubseteq\{(s, s),(t, t)\}=\alpha \alpha^{-1},
$$

we have $(\alpha, \beta) \notin \Omega$, and therefore $\Omega^{\prime} \backslash \Omega$ is nonempty. 
By the two lemmas above, we conclude that if $Y \subsetneq X$ and $|Y| \geq 3$, then the three partial orders $\subseteq \circ \leq, \Omega$ and $\Omega^{\prime}$ on $P T(X, Y)$ are different. Hence, we now have six distinct nontrivial partial orders on $P T(X, Y)$, with inclusions as indicated in the following diagram.

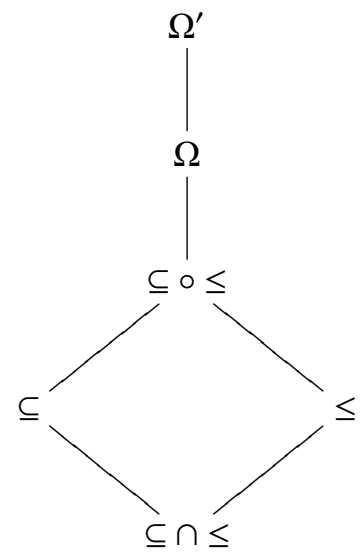

Next, we compare the partial orders on $I(X, Y)$. It is well known that the natural order and the subset order are the same on $I(X)$ [5, Proposition V.2.3], but in the semigroup $I(X, Y)$ we have the following result.

Theorem 4.4. For $\alpha, \beta \in I(X, Y), \alpha \leq \beta$ implies $\alpha \subseteq \beta$.

Proof. Let $\alpha, \beta \in I(X, Y)$ be such that $\alpha \leq \beta$. By Theorem 3.4, we have $\operatorname{dom} \alpha \subseteq$ $\operatorname{dom} \beta$, and for each $x \in \operatorname{dom} \alpha$ we have $x \alpha \in X \alpha \subseteq Y \beta$, which implies that $x \alpha=y \beta$ for some $y \in Y$, and hence $y \beta \in X \alpha$. Again by Theorem 3.4, we have $y \in \operatorname{dom} \alpha$ and $y \alpha=y \beta$. Hence $x \alpha=y \alpha$ and $x=y$, since $\alpha$ is injective. Thus $x \alpha=x \beta$ and therefore $\alpha \subseteq \beta$.

Hence the meet and join of these two partial orders on $I(X, Y)$ are $\leq$ and $\subseteq$ respectively. Next, we determine when these two relations on $I(X, Y)$ are equal.

Theorem 4.5. On $I(X, Y), \subseteq=\leq$ if and only if $X=Y$ or $|Y|=1$.

Proof. If $X=Y$, then $I(X, Y)=I(X)$, which implies that $\subseteq=\leq$ by [5, Proposition V.2.3]. If $|Y|=1$, let $Y=\{a\}$, and thus

$$
I(X, Y)=\left\{\left(\begin{array}{l}
x \\
a
\end{array}\right): x \in X\right\} \cup\{\emptyset\} .
$$

Hence, we see that if $\alpha, \beta \in I(X, Y)$ with $\alpha \subseteq \beta$, then $\alpha \leq \beta$. We conclude that $\subseteq=\leq$.

Now, suppose that $\subseteq=\leq$. If $Y \subsetneq X$ and $|Y|>1$, then there exist $a \in X \backslash Y$ and $b, c \in Y$ such that $b \neq c$. Define

$$
\alpha=\left(\begin{array}{l}
a \\
b
\end{array}\right), \quad \beta=\left(\begin{array}{ll}
a & b \\
b & c
\end{array}\right) .
$$


Then it is clear that $\alpha \subseteq \beta$. Since $\subseteq=\leq$, we obtain $\alpha \leq \beta$, from which it follows that $\{b\}=X \alpha \subseteq Y \beta=\{c\}$, which is a contradiction. Therefore, $X=Y$ or $|Y|=1$.

As with $P T(X, Y)$, the relations $\Omega^{\prime}$ and $\Omega$ defined at the beginning of Section 4 are also partial orders on $I(X, Y)$. In [11], the authors showed that $\Omega$ and $\Omega^{\prime}$ are equal on $I(X)$; thus, using the same proof as given for [11, Theorem 2.5], these two partial orders are also equal on $I(X, Y)$, and we also have $\subseteq \subseteq \Omega$. Finally, we characterise when $\Omega$ and $\subseteq$ are equal on $I(X, Y)$.

THeOrem 4.6. The partial order $\Omega=\subseteq$ on $I(X, Y)$ if and only if $|Y|=1$.

Proof. Suppose that $|Y|>1$. Then there are two distinct elements $x, y \in Y \subseteq X$. Define

$$
\alpha=\left(\begin{array}{l}
x \\
x
\end{array}\right), \quad \beta=\left(\begin{array}{ll}
x & y \\
y & x
\end{array}\right) .
$$

We can see that $(\alpha, \beta) \notin \subseteq$ but $(\alpha, \beta) \in \Omega^{\prime}=\Omega$.

Conversely, suppose that $|Y|=1$ and let $(\alpha, \beta) \in \Omega^{\prime}=\Omega$. If $\alpha=\emptyset$, then $\alpha \subseteq \beta$. However, if $\alpha \neq \emptyset$ then, since $|Y|=1$, we have $\alpha=\beta$. Hence $\alpha \subseteq \beta$ and $\Omega \subseteq \subseteq$. Similarly, we can show that $\subseteq \subseteq \Omega$.

From Theorems 4.5 and 4.6, we conclude that if $Y \subsetneq X$ and $|Y|>1$ then the three partial orders $\subseteq, \leq, \Omega$ are distinct.

\section{Compatible elements in the partial orders}

Let $\leq$ be a partial order on a semigroup $S$. An element $c \in S$ is said to be left (respectively, right) compatible if $c a \leq c b$ (respectively, $a c \leq b c$ ) for each $a, b \in S$. In this section, we characterise all elements in $P T(X, Y)$ and $I(X, Y)$ which are compatible with respect to $\leq$. We note that $\subseteq$ is always left and right compatible.

Lemma 5.1. Let $|Y|=1$ and $\alpha, \beta \in P T(X, Y)$. If $\alpha \leq \beta$, then $\alpha=\beta$ or $\alpha=\emptyset$.

Proof. Suppose that $\alpha \leq \beta$ and $\alpha \neq \emptyset$. Hence $1 \leq|X \alpha| \leq|Y|=1$, and then $X \alpha=Y$. For each $x \in \operatorname{dom} \beta$, we have $x \beta \in X \beta \subseteq Y=X \alpha$ and hence $x \in \operatorname{dom} \alpha$ and $x \alpha=x \beta$ by Theorem 3.3. Thus $\operatorname{dom} \beta \subseteq \operatorname{dom} \alpha$. Since $\alpha \leq \beta$, we have $\operatorname{dom} \alpha \subseteq \operatorname{dom} \beta$. Therefore, $\operatorname{dom} \alpha=\operatorname{dom} \beta$ which implies that $\alpha=\beta$.

By this lemma, we conclude that if $|Y|=1$, then every element in $P T(X, Y)$ and $I(X, Y)$ is left and right compatible with respect to $\leq$.

Theorem 5.2. Let $|Y|>1$ and $\gamma \in P T(X, Y)$.

(1) $\gamma$ is left compatible with $\leq$ if and only if $Y \gamma=Y$.

(2) $\gamma$ is right compatible with $\leq$ if and only if $\left(Y \subseteq \operatorname{dom} \gamma\right.$ and $\left.\gamma\right|_{Y}$ is injective) or $Y \cap \operatorname{dom} \gamma=\emptyset$. 
Proof. (1) Suppose that $Y \gamma \subsetneq Y$. Then there exists $y \in Y \backslash Y \gamma$. Choose $a, b \in Y \gamma$ and define $\alpha, \beta \in P T(X, Y)$ by

$$
\alpha=\left(\begin{array}{c}
\{b, y\} \\
y
\end{array}\right), \quad \beta=\left(\begin{array}{lll}
a & b & y \\
a & b & y
\end{array}\right) .
$$

Then $\alpha \leq \beta$. If $y \in X \gamma$, then $X \gamma \alpha=\{y\} \neq\{a, b, y\}=X \gamma \beta$, so $\gamma \alpha \neq \gamma \beta$. Also, if $y \notin X \gamma$, we then have $X \gamma \alpha=\{y\} \neq\{a, b\}=X \gamma \beta$, that is $\gamma \alpha \neq \gamma \beta$. Therefore, $\gamma \alpha \neq \gamma \beta$. Since $y \in X \gamma \alpha$ but $y \notin Y \gamma \beta$, we conclude that $\gamma \alpha \not \leq \gamma \beta$.

Conversely, assume that $Y \gamma=Y$. Let $\alpha, \beta \in P T(X, Y)$ be such that $\alpha \leq \beta$. Hence $X \gamma \alpha \subseteq X \alpha \subseteq Y \beta=Y \gamma \beta$ and

$$
\operatorname{dom} \gamma \alpha=(\operatorname{im} \gamma \cap \operatorname{dom} \alpha) \gamma^{-1} \subseteq(\operatorname{im} \gamma \cap \operatorname{dom} \beta) \gamma^{-1}=\operatorname{dom} \gamma \beta .
$$

Let

$$
(x, y) \in \operatorname{ker} \gamma \beta \cap(\operatorname{dom} \gamma \beta \times \operatorname{dom} \gamma \alpha) .
$$

Then $x \gamma \beta=y \gamma \beta, x \in \operatorname{dom} \gamma \beta$ and $y \in \operatorname{dom} \gamma \alpha$. Thus $x \gamma \in \operatorname{dom} \beta$ and $y \gamma \in \operatorname{dom} \alpha$, and hence

$$
(x \gamma, y \gamma) \in \operatorname{ker} \beta \cap(\operatorname{dom} \beta \times \operatorname{dom} \alpha) \subseteq \operatorname{ker} \alpha .
$$

Therefore, $x \gamma \alpha=y \gamma \alpha$ implies $(x, y) \in \operatorname{ker} \gamma \alpha$. For each $z \in \operatorname{dom} \gamma \beta$, if $z \gamma \beta \in X \gamma \alpha$, we have $z \gamma \beta \in X \alpha$; thus $z \gamma \in \operatorname{dom} \alpha$ (that is, $z \in \operatorname{dom} \gamma \alpha$ ) and $z \gamma \beta=z \gamma \alpha$. Therefore, $\gamma \alpha \leq \gamma \beta$.

(2) It is clear that if $Y \cap \operatorname{dom} \gamma=\emptyset$, then $\alpha \gamma=\emptyset=\beta \gamma$ for all $\alpha, \beta \in P T(X, Y)$. Assume that $Y \subseteq \operatorname{dom} \gamma$ and $\left.\gamma\right|_{Y}$ is injective. Let $\alpha, \beta \in P T(X, Y)$ be such that $\alpha \leq \beta$. Then $X \alpha \subseteq Y \beta$, which implies that $X \alpha \gamma \subseteq Y \beta \gamma$. Since $Y \subseteq \operatorname{dom} \gamma$,

$$
\begin{aligned}
\operatorname{dom} \alpha \gamma & =(\operatorname{im} \alpha \cap \operatorname{dom} \gamma) \alpha^{-1} \subseteq(Y \cap \operatorname{dom} \gamma) \alpha^{-1} \\
& =Y \alpha^{-1}=\operatorname{dom} \alpha \subseteq \operatorname{dom} \beta=(\operatorname{im} \beta \cap Y) \beta^{-1} \\
& \subseteq(\operatorname{im} \beta \cap \operatorname{dom} \gamma) \beta^{-1}=\operatorname{dom} \beta \gamma .
\end{aligned}
$$

Let $(x, y) \in \operatorname{ker} \beta \gamma \cap(\operatorname{dom} \beta \gamma \times \operatorname{dom} \alpha \gamma)$. Then $x \beta \gamma=y \beta \gamma, x \in \operatorname{dom} \beta \gamma \subseteq \operatorname{dom} \beta$ and $y \in \operatorname{dom} \alpha \gamma \subseteq \operatorname{dom} \alpha$. Since $\left.\gamma\right|_{Y}$ is injective, we obtain $x \beta=y \beta$, from which it follows that $(x, y) \in \operatorname{ker} \beta \cap(\operatorname{dom} \beta \times \operatorname{dom} \alpha) \subseteq \operatorname{ker} \alpha$ since $\alpha \leq \beta$. Hence, $x \alpha=y \alpha$ implies $x \alpha \gamma=y \alpha \gamma$, and so $(x, y) \in \operatorname{ker} \alpha \gamma$. For each $z \in \operatorname{dom} \beta \gamma$ with $z \beta \gamma \in X \alpha \gamma$, we have $z \beta \gamma=y \alpha \gamma$ for some $y \in X$. Since $\left.\gamma\right|_{Y}$ is injective, we have $z \beta=y \alpha \in X \alpha$, and thus $z \in \operatorname{dom} \alpha$ and $z \alpha=z \beta$. Hence

$$
z \in \operatorname{dom} \alpha=(\operatorname{im} \alpha \cap Y) \alpha^{-1} \subseteq(\operatorname{im} \alpha \cap \operatorname{dom} \gamma) \alpha^{-1}=\operatorname{dom} \alpha \gamma \quad \text { and } \quad z \alpha \gamma=z \beta \gamma .
$$

Therefore, $\alpha \gamma \leq \beta \gamma$.

Conversely, assume that $Y \cap \operatorname{dom} \gamma \neq \emptyset$ and $\left(\left.\gamma\right|_{Y}\right.$ is not injective or $\left.Y \nsubseteq \operatorname{dom} \gamma\right)$.

If $Y \cap \operatorname{dom} \gamma \neq \emptyset$ and $\left.\gamma\right|_{Y}$ is not injective, then there are $a, b \in Y \cap \operatorname{dom} \gamma$ such that $a \neq b$ and $a \gamma=b \gamma$. Define $\alpha, \beta \in P T(X, Y)$ by

$$
\alpha=\left(\begin{array}{l}
a \\
a
\end{array}\right), \quad \beta=\left(\begin{array}{ll}
a & b \\
a & b
\end{array}\right) .
$$


Hence, we obtain $\alpha \leq \beta$ by Theorem 3.3 and $\alpha \gamma \neq \beta \gamma$ since $b \in \operatorname{dom} \beta \gamma$ but $b \notin \operatorname{dom} \alpha \gamma$. We also have $\alpha \gamma \not \leq \beta \gamma$ since $(b, a) \in \operatorname{ker} \beta \gamma \cap(\operatorname{dom} \beta \gamma \times \operatorname{dom} \alpha \gamma)$ but $(b, a) \notin \operatorname{ker} \alpha \gamma$.

If $Y \cap \operatorname{dom} \gamma \neq \emptyset$ and $Y \nsubseteq \operatorname{dom} \gamma$, then there are $a \in Y \cap \operatorname{dom} \gamma$ and $b \in Y \backslash \operatorname{dom} \gamma$. Define $\alpha, \beta \in P T(X, Y)$ by

$$
\alpha=\left(\begin{array}{c}
\{a, b\} \\
a
\end{array}\right), \quad \beta=\left(\begin{array}{ll}
a & b \\
a & b
\end{array}\right) .
$$

Thus $\alpha \leq \beta$ and $\alpha \gamma \neq \beta \gamma$. Also, $\alpha \gamma \not \leq \beta \gamma$ since

$$
\operatorname{dom} \alpha \gamma=\{a, b\} \nsubseteq\{a\}=\operatorname{dom} \beta \gamma \text {. }
$$

Therefore, $\gamma$ is not right compatible.

It is clear that for each $\gamma \in I(X, Y)$ it is the case that dom $\gamma \subseteq Y$ if and only if $Y \gamma=X \gamma$. We use this fact to prove the following theorem.

Theorem 5.3. Let $|Y|>1$ and $\gamma \in I(X, Y)$. Then we have the following results.

(1) $\gamma$ is left compatible with respect to $\leq$ if and only if $\operatorname{dom} \gamma \subseteq Y$ or $\gamma$ is a constant map.

(2) $\gamma$ is always right compatible with respect to $\leq$.

Proof. (1) Suppose that $\operatorname{dom} \gamma \nsubseteq Y$ and $\gamma$ is not a constant map. Then $Y \gamma \subsetneq X \gamma$ and $|X \gamma|>1$, which implies that there exist $y \in X \gamma \backslash Y \gamma$ and $y \neq z \in X \gamma$. Define $\alpha, \beta \in I(X, Y)$ by

$$
\alpha=\left(\begin{array}{l}
y \\
y
\end{array}\right), \quad \beta=\left(\begin{array}{ll}
y & z \\
y & z
\end{array}\right) .
$$

We can see that $\alpha \leq \beta$. Since $X \gamma \alpha=\{y\} \neq\{y, z\}=X \gamma \beta$, we obtain $\gamma \alpha \neq \gamma \beta$. Since $y \in$ $X \gamma \alpha$ but $y \notin Y \gamma \beta$, we have $X \gamma \alpha \nsubseteq Y \gamma \beta$, from which it follows that $\gamma \alpha \not \leq \gamma \beta$. Therefore, $\gamma$ is not left compatible.

Conversely, let $\alpha, \beta \in I(X, Y)$ be such that $\alpha \leq \beta$. If dom $\gamma \subseteq Y$, then $Y \gamma=X \gamma$. Hence, we have $X \gamma \alpha \subseteq X \gamma \beta=Y \gamma \beta$ since $\alpha \subseteq \beta$ by Theorem 4.4 and

$$
\operatorname{dom} \gamma \alpha=(\operatorname{im} \gamma \cap \operatorname{dom} \alpha) \gamma^{-1} \subseteq(\operatorname{im} \gamma \cap \operatorname{dom} \beta) \gamma^{-1}=\operatorname{dom} \gamma \beta .
$$

Let $x \in \operatorname{dom} \gamma \beta$ and $x \gamma \beta \in X \gamma \alpha$. Then $(x \gamma) \beta \in X \alpha$, from which it follows that $x \gamma \alpha=$ $x \gamma \beta$ since $\alpha \leq \beta$. Therefore, $\gamma \alpha \leq \gamma \beta$. Now, if $\gamma$ is a constant map, then we can write $\gamma=\left(\begin{array}{l}x \\ a\end{array}\right)$. If $a \notin \operatorname{dom} \alpha$, then $\gamma \alpha=\emptyset \leq \gamma \beta$. If $a \in \operatorname{dom} \alpha$, then $a \in \operatorname{dom} \beta$ since $\operatorname{dom} \alpha \subseteq$ $\operatorname{dom} \beta$ and hence $\operatorname{dom} \gamma \alpha=\{x\}=\operatorname{dom} \gamma \beta$. Furthermore, $x \gamma \alpha=a \alpha=a \beta=x \gamma \beta$ since $\alpha \subseteq \beta$. Therefore, $\gamma \alpha=\gamma \beta$.

(2) Let $\alpha, \beta \in I(X, Y)$ be such that $\alpha \leq \beta$. Then $\alpha \subseteq \beta$, from which it follows that $\alpha \gamma \subseteq \beta \gamma$. Hence $\operatorname{dom} \alpha \gamma \subseteq \operatorname{dom} \beta \gamma$. Since $\alpha \leq \beta$, we have $X \alpha \gamma \subseteq Y \beta \gamma$. Let $x \beta \gamma \in X \alpha \gamma$. Then $x \beta \gamma=y \alpha \gamma$ for some $y \in X$. Since $\gamma$ is injective, we obtain $x \beta=y \alpha \in X \alpha$, which implies that $x \beta=x \alpha$. Hence $x \beta \gamma=x \alpha \gamma$, and therefore $\alpha \gamma \leq \beta \gamma$. 


\section{Maximal and minimal elements}

We aim now to find all maximal and minimal elements in $P T(X, Y) \backslash\{\emptyset\}$ with respect to the subset order. To do this, we first note that the empty set $\emptyset$ acts as a minimum with respect to $\subseteq$ and $\leq$ for $P T(X, Y)$.

Theorem 6.1. In $P T(X, Y) \backslash\{\emptyset\}$ with $\subseteq$, the following statements hold.

(1) $\alpha$ is minimal with respect to $\subseteq$ if and only if $|\operatorname{dom} \alpha|=1$.

(2) $\alpha$ is maximal with respect to $\subseteq$ if and only if $\operatorname{dom} \alpha=X$.

Proof. (1) If $|\operatorname{dom} \alpha|=1$, then for each $\beta \in P T(X, Y)$ with $\emptyset \neq \beta \subseteq \alpha$, it is clear that $\alpha=\beta$, and thus $\alpha$ is minimal with respect to $\subseteq$. If $|\operatorname{dom} \alpha|>1$, then there are $a, b \in \operatorname{dom} \alpha$ such that $a \neq b$. Define $\beta=\left(\begin{array}{c}a \\ a \alpha\end{array}\right)$, so $\emptyset \neq \beta \subsetneq \alpha$, which implies that $\alpha$ is not minimal.

(2) If $\operatorname{dom} \alpha=X$ and $\alpha \subseteq \beta$, then $X=\operatorname{dom} \alpha \subseteq \operatorname{dom} \beta \subseteq X$, from which it follows that $\alpha=\beta$, and hence $\alpha$ is maximal.

Conversely, assume that dom $\alpha \subsetneq X$. Write $\alpha=\left(\begin{array}{c}A_{i} \\ a_{i}\end{array}\right)$ and define

$$
\gamma=\left(\begin{array}{cc}
A_{i} & X \backslash \operatorname{dom} \alpha \\
a_{i} & a
\end{array}\right)
$$

for some $a \in Y$. It is clear that $\alpha \subsetneq \gamma$, so $\alpha$ is not maximal.

Next, we determine the maximal and minimal elements for $P T(X, Y)$ using the partial orders $\leq$.

Theorem 6.2. In $P T(X, Y) \backslash\{\emptyset\}$ with $\leq, \alpha$ is minimal if and only if $\alpha$ is a constant map or $Y \cap \operatorname{dom} \alpha=\emptyset$.

Proof. Let $\alpha, \gamma \in P T(X, Y)$ be such that $\emptyset \neq \gamma \leq \alpha$. If $\alpha$ is a constant map, then $X \alpha=$ $\{a\}$ for some $a \in Y$ and $X \gamma \subseteq Y \alpha=\{a\}$ from which it follows that $X \gamma=\{a\}$. Suppose that $\operatorname{dom} \gamma \subsetneq \operatorname{dom} \alpha$; then $|\operatorname{dom} \alpha| \geq 2$. Let $p, q \in \operatorname{dom} \alpha$ be such that $p \in \operatorname{dom} \gamma$ and $q \notin \operatorname{dom} \gamma$. We can see that $(q, p) \in \operatorname{ker} \alpha \cap(\operatorname{dom} \alpha \times \operatorname{dom} \gamma)$, but $(q, p) \notin \operatorname{ker} \gamma$, which is a contradiction. Thus $\operatorname{dom} \gamma=\operatorname{dom} \alpha$, and hence $\alpha=\gamma$. Now, if $Y \cap \operatorname{dom} \alpha=\emptyset$, then $\gamma<\alpha$ implies that $X \gamma \subseteq Y \alpha=\emptyset$, which contradicts $\gamma \neq \emptyset$, so we obtain $\alpha=\gamma$.

Conversely, assume that $\alpha$ is not a constant map and $Y \cap \operatorname{dom} \alpha \neq \emptyset$. Choose $a \in Y \cap \operatorname{dom} \alpha$ and define $\gamma: \operatorname{dom} \alpha \rightarrow\{a \alpha\}$. Since $|X \gamma|=1<|X \alpha|$, we obtain $\alpha \neq \gamma$. It is obvious that $X \gamma \subseteq Y \alpha$, dom $\gamma \subseteq \operatorname{dom} \alpha$ and

$$
\begin{aligned}
\operatorname{ker} \alpha \cap(\operatorname{dom} \alpha \times \operatorname{dom} \gamma) & =\operatorname{ker} \alpha \cap(\operatorname{dom} \gamma \times \operatorname{dom} \gamma) \\
& \subseteq \operatorname{dom} \gamma \times \operatorname{dom} \gamma=\operatorname{ker} \gamma .
\end{aligned}
$$

If $x \in \operatorname{dom} \alpha$ and $x \alpha \in X \gamma$, then $x \alpha=a \alpha=x \gamma$. Therefore, $\gamma<\alpha$ and $\alpha$ is not minimal.

Recall that $P F=\{\alpha \in P T(X, Y): X \alpha=Y \alpha\}$ and that when $Y=X$ we have $P F=P(X)$. 
Lemma 6.3. If $\alpha, \beta \in P T(X, Y)$ such that $\alpha \leq \beta$ and $\alpha \neq \beta$, then $\alpha \in P F$.

Proof. Let $\alpha, \beta \in P T(X, Y)$ be such that $\alpha \leq \beta$ and $\alpha \neq \beta$. Suppose that $\alpha \notin P F$. Then there exists $x \in X \backslash Y$ such that $x \alpha \notin Y \alpha$. Since $x \alpha \in X \alpha \subseteq Y \beta$, we have $x \alpha=y \beta$ for some $y \in Y$. Thus $y \beta=x \alpha \in X \alpha$ implies $y \in \operatorname{dom} \alpha$ and $y \alpha=y \beta$, and hence $x \alpha=y \alpha \in$ $Y \alpha$, which is a contradiction.

Since $|Y| \geq 1$, we then have $\emptyset$ is not maximal in $P T(X, Y)$.

Theorem 6.4. Let $\emptyset \neq \alpha \in P T(X, Y)$. Then $\alpha$ is maximal with respect to $\leq$ if and only if one of the following statements hold.

(1) $\alpha \notin P F$.

(2) $\alpha$ is surjective.

(3) $\alpha$ is injective and $\operatorname{dom} \alpha=X$.

Proof. If $Y=X$, then the theorem holds by [7, Theorem 14]. Now, we consider the case when $Y \subsetneq X$. Assume that $\alpha \in P F$ and $X \alpha \subsetneq Y$. Hence there is $a \in Y \backslash X \alpha$.

If $\alpha$ is not injective, then there exists $b \in Y$ such that $\left|b \alpha^{-1}\right|>1$. Choose $c \in b \alpha^{-1}$ if $b \alpha^{-1} \subseteq Y$, otherwise choose $c \in b \alpha^{-1} \backslash Y$. Define $\beta$ : dom $\alpha \rightarrow Y$ by

$$
z \beta= \begin{cases}z \alpha & \text { if } z \neq c, \\ a & \text { if } z=c .\end{cases}
$$

Hence $\alpha \neq \beta$, and it is clear that $\alpha, \beta$ satisfy conditions (1)-(3) of Theorem 3.3. Thus $\alpha<\beta$, and therefore $\alpha$ is not maximal.

If $\operatorname{dom} \alpha \subsetneq X$, then there exists $b \in X \backslash \operatorname{dom} \alpha$. Define $\beta: \operatorname{dom} \alpha \cup\{b\} \rightarrow Y$ by

$$
z \beta= \begin{cases}z \alpha & \text { if } z \in \operatorname{dom} \alpha \\ a & \text { if } z=b .\end{cases}
$$

Hence $\alpha \neq \beta$ and $\alpha<\beta$ since $\alpha, \beta$ satisfy conditions (1)-(3) of Theorem 3.3, so $\alpha$ is not maximal in $P T(X, Y)$.

Conversely, let $\beta \in P T(X, Y)$ be such that $\alpha \leq \beta$. If $\alpha \notin P F$, then $\alpha=\beta$ by Lemma 6.3. If $\alpha$ is surjective, then $x \beta \in Y=X \alpha$ for all $x \in \operatorname{dom} \beta$, and thus $x \in \operatorname{dom} \alpha$ and $x \alpha=x \beta$, and hence $\alpha=\beta$. Now, consider the case when $\alpha$ is injective and $\operatorname{dom} \alpha=X$. Since $\alpha \leq \beta$, we obtain $X=\operatorname{dom} \alpha \subseteq \operatorname{dom} \beta \subseteq X$ from which it follows that $\operatorname{dom} \beta=X=\operatorname{dom} \alpha$. For each $x \in X, x \alpha \in X \alpha \subseteq Y \beta$, that is, $x \alpha=y \beta$ for some $y \in Y$, which implies that $y \beta=x \alpha \in X \alpha$. Thus $y \in \operatorname{dom} \alpha$ and $y \alpha=y \beta$. Then $y \alpha=x \alpha$, from which it follows that $x=y$ since $\alpha$ is injective; hence $x \alpha=x \beta$ and therefore $\alpha=\beta$.

Theorem 6.5. Let $\alpha \in P T(X, Y)$. Then there exists a maximal element $\beta \in P T(X, Y)$ such that $\alpha \leq \beta$.

Proof. Suppose that $\alpha$ is not maximal. Then there are two cases. 
Case I: $\alpha$ is not injective. Then $\alpha \in P F, \alpha$ is not surjective and $\operatorname{dom} \alpha \subsetneq X$. Let $C(\alpha)=\left\{x \alpha^{-1}: x \in Y\right.$ and $\left.\left|x \alpha^{-1}\right|>1\right\}$. Since $\alpha$ is not injective, we have $C(\alpha)$ is nonempty. Since $\alpha \in P F$ and $\alpha$ is not surjective, we obtain $Y \alpha=X \alpha \subsetneq Y$, which implies that $Y \backslash X \alpha \neq \emptyset$. For each $C \in C(\alpha)$, we obtain $C=x \alpha^{-1}$ for some $x \in Y$ and $\left|x \alpha^{-1}\right|>1$. Since $x \in X \alpha=Y \alpha$, we have $x=y \alpha$ for some $y \in Y$. Thus $y \in C \cap Y$ since $y \in x \alpha^{-1}=C$. Hence $C \cap Y \neq \emptyset$; choose $d_{C} \in C \cap Y$. We can see that $C \backslash\left\{d_{C}\right\} \neq \emptyset$ since $|C|=\left|x \alpha^{-1}\right|>1$. We consider two subcases.

Subcase I:

$$
\left|\bigcup_{C \in C(\alpha)}\left(C \backslash\left\{d_{C}\right\}\right) \cup(X \backslash \operatorname{dom} \alpha)\right| \geq|Y \backslash X \alpha| .
$$

Then there is an injection

$$
\gamma: Y \backslash X \alpha \rightarrow \bigcup_{C \in C(\alpha)}\left(C \backslash\left\{d_{C}\right\}\right) \cup(X \backslash \operatorname{dom} \alpha) .
$$

For each $z \in \operatorname{im} \gamma$, we have $\left|z \gamma^{-1}\right|=1$, so let $z \gamma^{-1}=\left\{g_{z}\right\}$. Define $\beta \in P T(X, Y)$ by

$$
z \beta= \begin{cases}g_{z} & \text { if } z \in \operatorname{im} \gamma \\ z \alpha & \text { if } z \in \operatorname{dom} \alpha \backslash \operatorname{im} \gamma\end{cases}
$$

Then $\beta \neq \alpha$. To show that $\alpha<\beta$, let $x \in X \alpha=Y \alpha$. Then $x=y \alpha$ for some $y \in Y$. If $y \in \operatorname{dom} \alpha \backslash \operatorname{im} \gamma$, then $x=y \alpha=y \beta \in Y \beta$. If $y \in \operatorname{im} \gamma$, then

$$
y \in \bigcup_{C \in C(\alpha)}\left(C \backslash\left\{d_{C}\right\}\right) \cup(X \backslash \operatorname{dom} \alpha) .
$$

It is clear that $y \in \operatorname{dom} \alpha$, and hence $y \in C \backslash\left\{d_{C}\right\}$ for some $C \in C(\alpha)$. Since $y \in x \alpha^{-1}$, we conclude that $C=x \alpha^{-1}$. Thus $x=d_{C} \alpha=d_{C} \beta \in Y \beta$ since $d_{C} \notin \operatorname{im} \gamma$. Hence $X \alpha \subseteq Y \beta$. It is clear that $\operatorname{dom} \alpha \subseteq \operatorname{dom} \beta$. Let $(x, y) \in \operatorname{ker} \beta \cap(\operatorname{dom} \beta \times \operatorname{dom} \alpha)$. Then $x \beta=y \beta, x \in$ $\operatorname{dom} \beta$ and $y \in \operatorname{dom} \alpha$. Thus by the definition of $\beta$, we have $x, y \in \operatorname{im} \gamma$ or $x, y \notin \operatorname{im} \gamma$. If $x, y \in \operatorname{im} \gamma$, then $g_{x}=x \beta=y \beta=g_{y}$, which implies that $x \gamma^{-1}=\left\{g_{x}\right\}=\left\{g_{y}\right\}=y \gamma^{-1}$. Hence $x=y$ since $\gamma$ is injective. Therefore, $x=y \in \operatorname{dom} \alpha$ and $x \alpha=y \alpha$. If $x, y \notin$ $\operatorname{im} \gamma$, then $x, y \in \operatorname{dom} \alpha \backslash \operatorname{im} \gamma$. Hence $x \alpha=x \beta=y \beta=y \alpha$; that is, $(x, y) \in \operatorname{ker} \alpha$. Let $x \in \operatorname{dom} \beta$ and $x \beta \in X \alpha$. Thus, $x \in \operatorname{dom} \alpha \backslash \operatorname{im} \gamma$ (for if $x \in \operatorname{im} \gamma$, then $x \beta=g_{x} \in Y \backslash X \alpha$, which is a contradiction), from which it follows that $x \alpha=x \beta$. Therefore, $\alpha<\beta$.

To show that $\beta$ is surjective, let $y \in Y$. If $y \in Y \backslash X \alpha$, then $y \gamma \in \operatorname{im} \gamma$, so we obtain $(y \gamma) \beta=g_{y \gamma} \in(y \gamma) \gamma^{-1}=\{y\}$ since $\gamma$ is injective. Hence $y=(y \gamma) \beta$. Now, if $y \in X \alpha$, then $y=x \alpha$ for some $x \in X$. If $x \in \operatorname{im} \gamma$, then $x \in C$ for some $C \in C(\alpha)$ and $y=x \alpha=d_{C} \alpha=$ $d_{C} \beta$. If $x \notin \operatorname{im} \gamma$, then $y=x \alpha=x \beta$. Therefore, $\beta$ is maximal by Theorem 6.4.

Subcase II:

$$
\left|\bigcup_{C \in C(\alpha)}\left(C \backslash\left\{d_{C}\right\}\right) \cup(X \backslash \operatorname{dom} \alpha)\right|<|Y \backslash X \alpha| .
$$


Then there exists an injection

$$
\gamma: \bigcup_{C \in C(\alpha)}\left(C \backslash\left\{d_{C}\right\}\right) \cup(X \backslash \operatorname{dom} \alpha) \rightarrow Y \backslash X \alpha .
$$

Define $\beta \in P T(X, Y)$ by

$$
z \beta= \begin{cases}z \gamma & \text { if } z \in \operatorname{dom} \gamma \\ z \alpha & \text { if } z \in \operatorname{dom} \alpha \backslash \operatorname{dom} \gamma\end{cases}
$$

Then $\alpha \neq \beta$. Let $x \in X \alpha=Y \alpha$. Then $x=y \alpha$ for some $y \in Y$. If $y \in \operatorname{dom} \alpha \backslash \operatorname{dom} \gamma$, then $x=y \alpha=y \beta \in Y \beta$. If $y \in \operatorname{dom} \gamma$, then

$$
y \in \bigcup_{C \in C(\alpha)}\left(C \backslash\left\{d_{C}\right\}\right) \cup(X \backslash \operatorname{dom} \alpha) .
$$

Since $y \in \operatorname{dom} \alpha$, we obtain $y \in C \backslash\left\{d_{C}\right\}$ for some $C \in C(\alpha)$. Since $y \in x \alpha^{-1}$, so $C=x \alpha^{-1}$. Hence $x=d_{C} \alpha=d_{C} \beta \in Y \beta$. Thus $X \alpha \subseteq Y \beta$. It is clear that $\operatorname{dom} \alpha \subseteq \operatorname{dom} \beta$. Let $(x, y) \in \operatorname{ker} \beta \cap(\operatorname{dom} \beta \times \operatorname{dom} \alpha)$. Then $x \beta=y \beta, x \in \operatorname{dom} \beta$ and $y \in \operatorname{dom} \alpha$. Hence, by the definition of $\beta$, we conclude that $x, y \in \operatorname{dom} \gamma$ or $x, y \in \operatorname{dom} \alpha \backslash \operatorname{dom} \gamma$. If $x, y \in \operatorname{dom} \gamma$, then $x \gamma=x \beta=y \beta=y \gamma$, which implies that $x=y$ since $\gamma$ is injective. Hence $x=y \in \operatorname{dom} \alpha$ and $x \alpha=y \alpha$. If $x, y \in \operatorname{dom} \alpha \backslash \operatorname{dom} \gamma$, then $x \alpha=x \beta=y \beta=y \alpha$. Thus $(x, y) \in \operatorname{ker} \alpha$. Let $x \in \operatorname{dom} \beta$ and $x \beta \in X \alpha$. If $x \in \operatorname{dom} \gamma$, then $x \beta=x \gamma \in Y \backslash X \alpha$, which is a contradiction. Hence $x \notin \operatorname{dom} \gamma$; so $x \in \operatorname{dom} \alpha \backslash \operatorname{dom} \gamma$, from which it follows that $x \beta=x \alpha$. Therefore, $\alpha<\beta$.

To show that $\beta$ is injective, let $x, y \in \operatorname{dom} \beta$ be such that $x \beta=y \beta$. Then $x, y \in \operatorname{dom} \gamma$ or $x, y \in \operatorname{dom} \alpha \backslash \operatorname{dom} \gamma$ by the definition of $\beta$. If $x, y \in \operatorname{dom} \gamma$, then $x=y$ by the same proof as given above. If $x, y \in \operatorname{dom} \alpha \backslash \operatorname{dom} \gamma$, then $x \alpha=y \alpha$ (see above). Let $x \alpha=z=y \alpha$. Then $x, y \in z \alpha^{-1}$ implies $z \alpha^{-1}=C$ for some $C \in C(\alpha)$. We conclude that $x=d_{C}=y$. We can see that $\operatorname{dom} \beta=\operatorname{dom} \alpha \cup \operatorname{dom} \gamma=X$. Therefore, $\beta$ is maximal by Theorem 6.4.

Case II: $\alpha$ is injective. Then $\alpha \in P F, \alpha$ is not surjective and $\operatorname{dom} \alpha \subsetneq X$. Hence $Y \backslash X \alpha \neq \emptyset \neq X \backslash \operatorname{dom} \alpha$.

Subcase I: $\mid X \backslash$ dom $\alpha|\geq| Y \backslash X \alpha \mid$. Then there is an injection

$$
\gamma: Y \backslash X \alpha \rightarrow X \backslash \operatorname{dom} \alpha
$$

For each $z \in \operatorname{im} \gamma$, we have $\left|z \gamma^{-1}\right|=1$ since $\gamma$ is injective, so let $z \gamma^{-1}=\left\{g_{z}\right\}$. Define $\beta \in P T(X, Y)$ by

$$
z \beta= \begin{cases}g_{z} & \text { if } z \in \operatorname{im} \gamma, \\ z \alpha & \text { if } z \in \operatorname{dom} \alpha .\end{cases}
$$

We see that $\alpha \neq \beta$. Let $x \in X \alpha=Y \alpha$. Then $x=y \alpha$ for some $y \in Y$, which implies that $y \in \operatorname{dom} \alpha$. Thus $x=y \alpha=y \beta \in Y \beta$. That is, $X \alpha \subseteq Y \beta$. It is clear that $\operatorname{dom} \alpha \subseteq \operatorname{dom} \beta$. 
Let $(x, y) \in \operatorname{ker} \beta \cap(\operatorname{dom} \beta \times \operatorname{dom} \alpha)$. Then $x \beta=y \beta, x \in \operatorname{dom} \beta$ and $y \in \operatorname{dom} \alpha$. If $x \in \operatorname{im} \gamma$, then $y \alpha=y \beta=x \beta=g_{x} \in Y \backslash X \alpha$ (since $y \in \operatorname{dom} \alpha$ ), which is a contradiction. Hence $x \in \operatorname{dom} \alpha$ and $x \alpha=x \beta=y \beta=y \alpha$. Thus $(x, y) \in \operatorname{ker} \alpha$. Let $x \in \operatorname{dom} \beta$ and $x \beta \in X \alpha$. Then $x \in \operatorname{dom} \alpha$ and $x \alpha=x \beta$. Hence $\alpha<\beta$.

To show that $\beta$ is surjective, let $y \in Y$. If $y \in Y \backslash X \alpha$, then by the same proof as given for case I (subcase I), we have $y=(y \gamma) \beta$. If $y \in X \alpha$, then $y=x \alpha$ for some $x \in X$. Hence $y=x \alpha=x \beta$. Therefore, $\beta$ is maximal by Theorem 6.4.

Subcase II: $|X \backslash \operatorname{dom} \alpha|<|Y \backslash X \alpha|$. Then there is an injection

$$
\gamma: X \backslash \operatorname{dom} \alpha \rightarrow Y \backslash X \alpha .
$$

Define $\beta \in P T(X, Y)$ by

$$
z \beta= \begin{cases}z \gamma & \text { if } z \in \operatorname{dom} \gamma, \\ z \alpha & \text { if } z \in \operatorname{dom} \alpha .\end{cases}
$$

Therefore, $\alpha \neq \beta$. Let $x \in X \alpha=Y \alpha$. Then $x=y \alpha$ for some $y \in Y$, which implies that $y \in \operatorname{dom} \alpha$. Hence $x=y \alpha=y \beta \in Y \beta$. Thus $X \alpha \subseteq Y \beta$. It is clear that $\operatorname{dom} \alpha \subseteq \operatorname{dom} \beta$. Let

$$
(x, y) \in \operatorname{ker} \beta \cap(\operatorname{dom} \beta \times \operatorname{dom} \alpha) .
$$

Then $x \beta=y \beta, x \in \operatorname{dom} \beta$ and $y \in \operatorname{dom} \alpha$. If $x \in \operatorname{dom} \gamma$, then $y \alpha=y \beta=x \beta=x \gamma \in$ $Y \backslash X \alpha$, which is a contradiction. Hence $x \in \operatorname{dom} \alpha$ and $x \alpha=x \beta=y \beta=y \alpha$. Thus $(x, y) \in \operatorname{ker} \alpha$. Let $x \in \operatorname{dom} \beta$ and $x \beta \in X \alpha$. If $x \in \operatorname{dom} \gamma$, then $x \beta=x \gamma \in Y \backslash X \alpha$, which is a contradiction. Therefore, $x \in \operatorname{dom} \alpha$ and $x \alpha=x \beta$. Hence $\alpha<\beta$.

To show that $\beta$ is injective, let $x, y \in \operatorname{dom} \beta$ be such that $x \beta=y \beta$. If $x, y \in \operatorname{dom} \gamma$, then $x=y$ since $\gamma$ is injective. If $x, y \notin \operatorname{dom} \gamma$, then $x, y \in \operatorname{dom} \alpha$, which implies that $x \alpha=x \beta=y \beta=y \alpha$. Since $\alpha$ is injective, $x=y$. We can see that $\operatorname{dom} \beta=\operatorname{dom} \alpha \cup$ $\operatorname{dom} \gamma=X$. Therefore, $\beta$ is maximal by Theorem 6.4 .

We end this section with the following remark.

REMARK 6.6. We believe that with some mild modifications of the proofs which are given for $P T(X, Y)$, we obtain all maximal and minimal elements for $I(X, Y)$ with respect to the orders $\leq$ and $\subseteq$.

\section{Acknowledgement}

The authors wish to thank the referee for the careful review, which helped to improve the readability of this paper.

\section{References}

[1] A. H. Clifford and G. B. Preston, The Algebraic Theory of Semigroups, Vol. 1, Mathematical Surveys, 7 (American Mathematical Society, Providence, RI, 1961).

[2] V. H. Fernandes and J. Sanwong, 'On the ranks of semigroups of transformations on a finite set with restricted range', Algebra Colloq., to appear. 
[3] R. E. Hartwig, 'How to partially order regular elements', Math. Jap. 25(1) (1980), 1-13.

[4] J. M. Howie, "Naturally ordered bands", Glasg. Math. J. 8 (1967), 55-58.

[5] J. M. Howie, An Introduction to Semigroup Theory (Academic Press, London, 1976).

[6] G. Kowol and H. Mitsch, 'Naturally ordered transformation semigroups', Monatsh. Math. 102 (1986), 115-138.

[7] M. P. O. Marques-Smith and R. P. Sullivan, 'Partial orders on transformation semigroups', Monatsh. Math. 140 (2003), 103-118.

[8] H. Mitsch, 'A natural partial order for semigroups', Proc. Amer. Soc. 97(3) (1986), 384-388.

[9] K. S. S. Nambooripad, 'The natural partial order on a regular semigroup', Proc. Edinb. Math. Soc. (2) 23 (1980), 249-260.

[10] J. Sanwong and W. Sommanee, 'Regularity and Green's relations on a semigroup of transformation with restricted range', Int. J. Math. Math. Sci. 2008 (2008), Art. ID 794013, 11 pp.

[11] B. Singha, J. Sanwong and R. P. Sullivan, 'Partial orders on partial Baer-Levi semigroups', Bull. Aust. Math. Soc. 81 (2010), 195-207.

[12] J. S. V. Symons, 'Some results concerning a transformation semigroup', J. Aust. Math. Soc. (Series A) 19 (1975), 413-425.

[13] V. Vagner, 'Generalized groups', Dokl. Akad. Nauk SSSR 84 (1952), 1119-1122 (in Russian).

KRITSADA SANGKHANAN, Department of Mathematics, Faculty of Science, Chiang Mai University, Chiangmai 50200, Thailand

e-mail: kritsada_kst@hotmail.com

JINTANA SANWONG, Department of Mathematics, Faculty of Science, Chiang Mai University, Chiangmai 50200, Thailand

and

Material Science Research Center, Faculty of Science,

Chiang Mai University, Thailand

e-mail: jintana.s@cmu.ac.th 